\title{
A Case of Isolated Cardiac Lipidosis
}

\author{
C. F. ROSS AND E. M. BELTON \\ From the North West Surrey Group Pathology Laboratory and Department of Paediatrics, St. Peter's Hospital, \\ Chertsey, Surrey
}

The heart may be involved in certain lipidoses as part of a generalized disorder, particularly in the xanthomatoses, but as far as we can ascertain from a search of the published reports no case of isolated lipidosis of the heart has hitherto been described.

\section{Case History}

A baby girl, aged 16 months, the youngest child of healthy parents with three healthy sibs aged 7,4 , and 3 years, was admitted to St. Peter's Hospital, Chertsey, in August 1962. Until 48 hours before admission her progress had been entirely satisfactory, with normal milestones and no serious illnesses. She then became fretful and pale and repeatedly vomited. She was seen by her doctor 24 hours later and was admitted to hospital the next morning.

On admission, she was a small well-nourished child, pale and tired but not acutely ill. Her pulse rate was very rapid-about 300 a minute, though regular. The heart was a little enlarged but there was no evidence of failure. Carotid compression produced a fall in pulse rate to about 140 a minute, but it rose again on releasing the pressure. An electrocardiogram showed atrial tachycardia at a regular rate of 300 a minute, but no other abnormal features. She was sedated and digitalized, and the pulse rate fell to about 170 a minute after 7 hours. She was kept on digitalis and sedatives for several days and the drugs were then gradually withdrawn. On the 8th day after admission there was another episode of tachycardia of sudden onset which again responded to similar treatment, though more slowly, and she was kept on digitalis. In spite of this she suffered further prolonged attacks, and quinidine was then tried. This was successful but had to be given at increasingly short intervals and was accompanied by vomiting. The heart began to dilate and the liver to enlarge. She was seen in consultation by Dr. R. K. Brown (Queen Elizabeth Hospital for Children, London) who confirmed the findings and could find no pointers to serious underlying disease. Six weeks after admission, during a period of prolonged tachycardia, she suddenly collapsed with cardiac arrest. The heart was started after three minutes' external cardiac massage and spontaneous beating established by open massage. She thereafter improved for a time, though with recurrent attacks of tachycardia, but succumbed to death 8 weeks after admission in an episode of cardiorespiratory arrest with no response to treatment.

\section{Pathology}

Necropsy was performed 12 hours after death. Apart from cerebral oedema, the only abnormal findings were in the heart. The latter weighed $80 \mathrm{~g}$. and showed a slightly milky epicardium in which were a few petechiae. The pericardial sac was normal. There was no congenital malformation and the great vessels were normal. The ductus arteriosus and foramen ovale were closed. There was some slight endocardial thickening in the neighbourhood of the latter. The tricuspid and pulmonary valves were normal. There was a yellowishbrown lobulated nodule $(4 \mathrm{~mm}$. diam.) in the base of the mitral valve towards the medial junction of the cusps. Both the latter were thicker than normal and in the base of the anterior cusp were several small yellowish-brown plaque-like thickenings visible on both surfaces and extending into the adjacent endocardium at the base of the septum, just reaching the base of the right coronary cusp of the aortic valve. The latter was normal apart from this. The coronary arteries were of normal size and distribution. The myocardium was generally pale but firm (Fig. 1).

Sections of formalin-fixed material were made of heart, lung, liver, spleen, kidneys, pancreas, adrenals, pituitary, thymus, ileum, and cerebrum. The latter showed changes due to anoxia but no evidence of any sort of adventitious material and no relevant changes were demonstrable in any of the other organs except the heart. Sections through the affected parts showed infiltration by numerous cells which were about $50 \mu$ diameter and distended with vacuoles and granular material, the intervening tissue being somewhat fibrous (Fig. 2). The material stained blue with alcian blue and was partly periodic acid-Schiff positive and stained lightly with Best's carmine for glycogen. Frozen sections revealed much isotropic lipid which accounted for the bulk of the adventitious material. Other parts of the myocardium were normal. Frozen sections of cerebrum, 


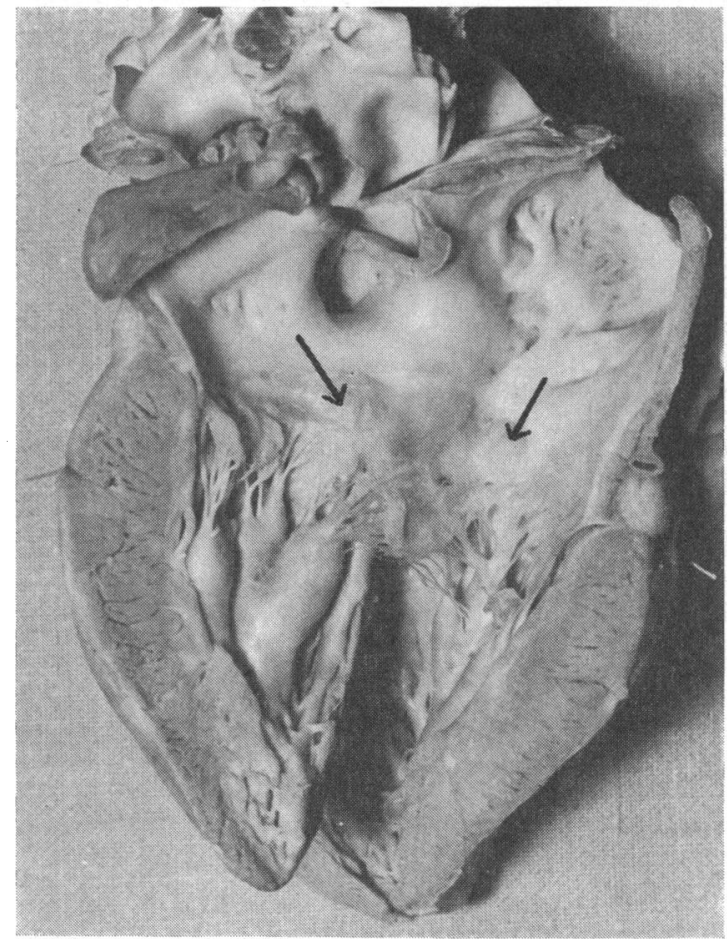

FIG. 1.-Heart showing thickenings in base of mitral valve (arrows) $(\times 1 \cdot 5)$. liver, and spleen revealed no abnormal material. The heart was sent to Professor R. E. B. Hudson (National Heart Hospital, London) who examined the conducting system and reported that the sinu-atrial node was infiltrated with lipoprotein-laden cells (Fig. 3). Dr. I. M. P. Dawson (Vincent Square Laboratories of the Westminster Hospital, London) undertook the histochemical examination of selected portions of the affected tissue and reported that the adventitious material was a complex lipid insoluble in ordinary fat solvents. Unfortunately the amount of material available did not permit of a full analysis.

\section{Discussion}

The lesion appears to be a congenital storage disorder confined to one small part of the heart. The onset of symptoms was presumably delayed until a sufficient amount of the abnormal substance had accumulated in the affected region. In most lipidoses many organs are involved, though in the amaurotic family idiocies the abnormal material is often confined to the nervous system. There is no a priori reason why, therefore, abnormal material may not be confined to a single system, in the present case the heart.

Recurrent paroxysmal tachycardia is not uncommon in childhood but usually responds well to simple measures such as vagal stimulation. Car-

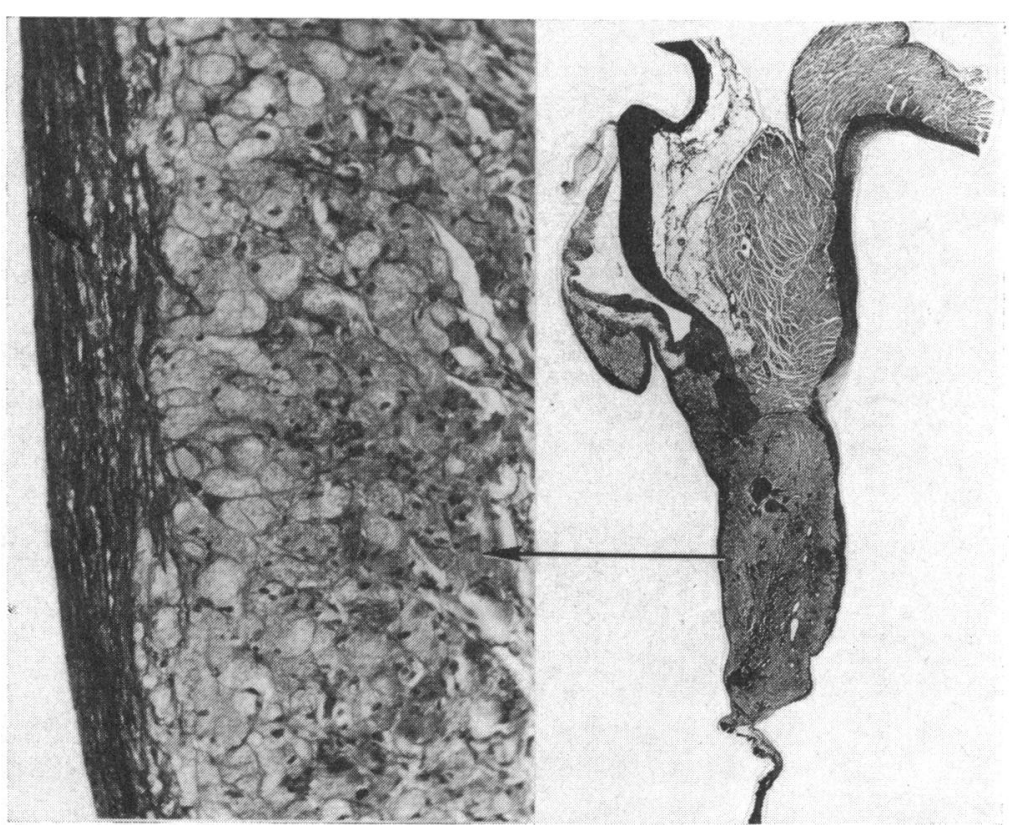

FIG. 2.-Section through atria, aorta, aortic and mitral valves (on right, $\times 3.6$ ), with photomicrograph of lipoprotein-laden cells infiltrating the latter (Verhoeff-van Gieson, $\times 124$ ). 


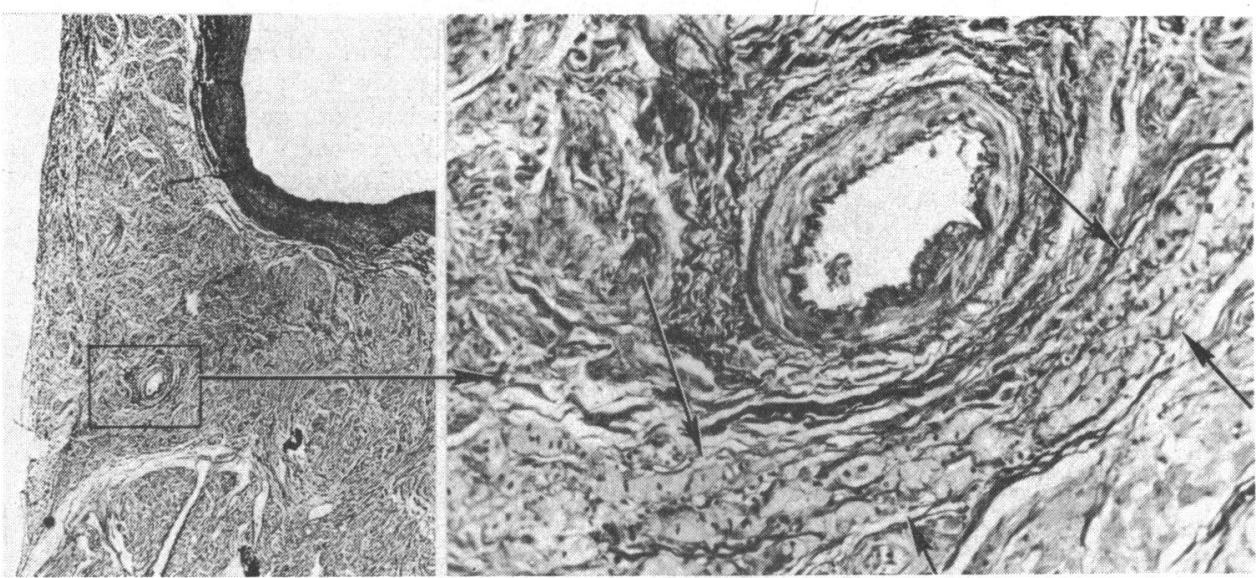

Frg. 3.-Section through sinu-atrial node showing infiltration by lipoprotein-laden cells (between arrows) ( $x 18$ on left, $\times 136$ on right).

diac failure is likely to supervene if the heart rate is not controlled within about 36 hours, especially in infants. About 5 per cent of children suffering repeated attacks show electrocardiographic evidence of Wolff-Parkinson-White syndrome, but in the vast majority there is no detectable abnormality between attacks. Dr. R. Bonham Carter (1962, personal communication) has said that in those rare cases which fail to respond to classical treatment, some intracardiac disease-usually a rhabdomyomais almost always found.

\section{Summary}

The case of a 16-month-old female infant is described, who presented with tachycardia which did not respond to usual measures and at necropsy was found to have a lipidosis confined to the base of the heart and involving the conduction system.

We are greatly indebted to Professor R. E. B. Hudson for his examination of the conducting system and photomicrographs, to Dr. I. M. P. Dawson for his histochemical studies, to Dr. R. K. Brown for seeing the patient, and to Dr. R. Bonham Carter for clinical advice. 